\title{
Differential inertia of lower and upper parts of a skyscraper drives swaying of a building
}

Lipeng Liao ${ }^{1}$, Chanyuk Lam ${ }^{1}$, Yuhan Lin ${ }^{1}$, Jingli Peng ${ }^{1}$, Jess Lan Ouyang ${ }^{1}$, Man Tang ${ }^{1}$, Qiuyun Liu ${ }^{1, *}$

${ }^{1}$ School of Life Sciences, Sun Yat-Sen University, Guangzhou 510275, China.

*Correspondence author: Qiuyun Liu, School of Life Sciences, Sun Yat-sen University, Guangzhou 510275, China.

E-mail address: liuqiuyunsysu@163.com (Q. Liu) 


\begin{abstract}
The lower part of a skyscraper possesses enormous weight giving the gravity of the total building, whereas the upper part is smaller in mass. Upon a gust of wind or other factors, the lower part accelerates and decelerates more slowly than the upper part, and then the upper part moves backwards first and then forwards. It resembles a traveling bus, the standing passengers move backwards and forwards upon vehicle starting from standing-still or a sudden stop.
\end{abstract}

\title{
Keywords
}

differential inertia; lower and upper parts of a skyscraper; swaying; accelerates; decelerate 
Shaking of skyscrapers is no stranger to city dwellers. So it is not surprising that the 1,000-foot SEG Plaza in Shenzhen, south China started to sway on one afternoon this past week. What causes such shaking for high-rise buildings across the world?

To find answers, we need to turn attention to the emerging theory of seismicity (1-18). According to Newton's Law of Inertia, larger mass accelerates and decelerates more slowly than smaller object, and smaller mass accelerates and decelerates faster than larger object (1-18). The earth self-rotates and revolves around the Sun, collectively it accounts for the northeastward drift of the Tibetan Plateau upon the impact of solar and lunar gravitational pulls (1-18). This theory also explains the swaying of Humen Bridge and other bridges (19-20).

Considering a high-rise building, the lower part is very heavy giving enormous gravity, whereas the upper part is smaller in mass. Upon a gust of wind, the lower part accelerates and decelerates more slowly than the upper part, and immediately after the upper part moves backwards first, and then forwards. It is like a traveling bus, the standing passengers move backwards and forwards upon starting or abrupt stop.

\section{Conflict of interest}

None declared.

\section{ACKNOWLEDGMENT}

This work was supported by the Science and Technology Transformation Program of Sun Yat-sen University of China (33000-18843234) and Guangzhou Science and Technology Program (201804010328) to Q. Liu. We thank Yan Shi for editing.

\section{References}

1. Yan $\mathrm{S}, \mathrm{Du}, \mathrm{Y}, \mathrm{Xu} \mathrm{H}$, et al. Filtering out non-specific animal behavior to better predict impending earthquakes. OSF Preprints. 2020. https://doi.org/10.31219/osf.io/bs3qd.

2. Wan Y, Shi Y, Li Z, et al. How focal depth is generated? Science 2017; http://science.sciencemag.org/content/354/6315/1027/tab-e-letters.

3. Du Y, Hu X, Li Z, Liu Q. How pre-earthquake atmospheric thermal energy is generated? Science 2017; http://science.sciencemag.org/content/354/6315/1027/tab-e-letters.

4. Weng S, Reina S, Wang K, et al. Supplement to earthquake prediction based on Voltage-Dependent Anion Channels. Science 2017; http://science.sciencemag.org/content/354/6308/88/tab-e-letters.

5. Du Y, He J, Pinto VD, et al. Earthquake prediction based on Voltage-Dependent Anion Channels. Science 2017; http://science.sciencemag.org/content/354/6308/88/tab-e-letters.

6. Varga P, Gambis D, Bus Z, Bizouard C. Fundamental astronomy: new concepts and models for high accuracy observations. Paris: Observatoire de Paris 2005;115-120.

7. Yan S, Li J, Liu Q. Earthquakes-an intricate trio dance of gravitation. Science 2018; http://science.sciencemag.org/content/354/6315/1027/tab-e-letters.

8. Yan S, Wang X, Qi J, Liu Q. Volcanoes or earthquakes: Wrist wrestling. Science, 2019; http://science.sciencemag.org/content/358/6370/1520/tab-e-letters.

9. Kilston S, Knopoff L. Lunar-solar periodicities of large earthquakes in southern California. Nature 
1983;304(5921):21-25.

10. He R, He S, Nuertai X, et al. The faster acceleration of smaller masses in the west of Americas. Science 2017; http://science.sciencemag.org/content/354/6315/1027/tab-e-letters.

11. Yang K, Xu H, Liu W, Liu Q. Why several megaquakes strike around noon? Science 2019; https://science.sciencemag.org/content/354/6308/88/tab-e-letters.

12. Yan $\mathrm{S}, \mathrm{Xu} \mathrm{X}, \mathrm{Chen} \mathrm{L}$, et al. Earthquakes: The rocking cradle. Science 2019; https://science.sciencemag.org/content/354/6315/1027/tab-e-letters.

13. Kolvankar VG, Deshpande SS, Manjre AS, et al. Lunar Periodicities and earthquakes. New Concepts Global Tectonics Newsl 2010;56:32-49.

14. Xu H, Wu J, Liu Q. Earth's self-rotation: high-velocity movements manifested in the solar and lunar gravitational fields. Science 2019; https://science.sciencemag.org/content/354/6308/88/tab-eletters.

15. Liu Q, Gao J, Pan W, et al. Differentia in decelerations of masses: when the Earth meets lunar and solar pulls. Science 2017; http://science.sciencemag.org/content/354/6315/1027/tab-e-letters.

16. Yang K, Liu J, Liu Q. The basis for continually-distributing daily seismic activities. Science 2019; https://science.sciencemag.org/content/354/6308/88/tab-e-letters).

17. Zhong H, Wang K, Shi Y, et al. Tsunamis is a prime example of the vertical component of the $\begin{array}{llll}\text { forces during 2017; } & \text { Science }\end{array}$ http://science.sciencemag.org/content/358/6367/1164/tab-e-letters.

18. De Pinto V, Reina S, Wan Y, et al. Why burrowing animals are highly sensitive toward impending earthquakes? Science 2017; http://science.sciencemag.org/content/356/6340/841/tab-e-letters.

19. Ran H, Lu X, Zheng R, et al. Vortex-induced vibration: The slower deceleration of the west bank leads to the shortening of the Humen Bridge in Guangdong, China. OSF Preprints. 2020. doi:10.31219/osf.io/3t4a6. https://osf.io/3t4a6

20. Lu X, Ran H, Zheng R, et al. 2020. The revolution of the earth around the sun causes the vortexinduced vibration of south-north trending bridges and impacts seismic activities. OSF Preprints. 2020. https://doi.org/10.31219/osf.io/ycjhz 